\title{
SIMPLE DEVICES FOR THE PHYSICALLY DISABLED
}

\author{
By Ernest Harrison, Jr., Ph.D. and Guy T. Vise, Jr., M.D.* \\ Biomedical Engineer and Chief Executive Officer/Medical Director, Mississippi \\ Methodist Rehabilitation Center, I 350 East Woodrow Wilson, Fackson, Mississippi \\ 392I6., U.S.A.
}

Summary. A report is given on a number of devices for the disabled that have been developed in the Biomedical Engineering Department at the Mississippi Methodist Rehabilitation Center. The primary criteria used in selection of devices to be included use simplicity, ease of fabrication and low cost.

Key words: Physically disabled; Spinal paralysis; Assistive devices.

\section{Introduction}

DISABLED PERSONS represent a significant portion of the population and orthopaedic impairment represents one of the larger categories (Fig. I). The prevalence of the use of special ambulatory aids was assessed by the National Centre for Health Statistics (Fig. 2).

With such a large population of disabled requiring assistive devices, an industry has developed to provide these special aids to the handicapped. Assistive devices range from the very simple and very inexpensive to the extremely complex and very expensive. This paper illustrates some simple devices which can be made within the local environment often by members of the family or by relatively unskilled personnel. Complicated electronic and mechanical devices, although absolutely necessary in certain applications, are not considered in this paper.

\section{Discussion of devices}

An ideal assistive device is defined to have the following characteristics:

I. it must provide the desired function;

2. it should be of low cost;

3. it should have high reliability;

4. it should be easy to use;

5. it should require minimal training;

6. ideally devices that are worn on the patient during ambulation or during movement, should be lightweight. The devices reported here were developed at the Mississippi Methodist Rehabilitation Center to meet the needs of its patients. The primary criterion used in selecting the devices for this paper is that fabrication of the device must be easy, inexpensive and within the capabilities of relatively unskilled personnel.

\footnotetext{
* Part of a paper read at the Annual Scientific Meeting of the International Medical Society of Paraplegia, Athens, Greece, Oct. I982.
} 


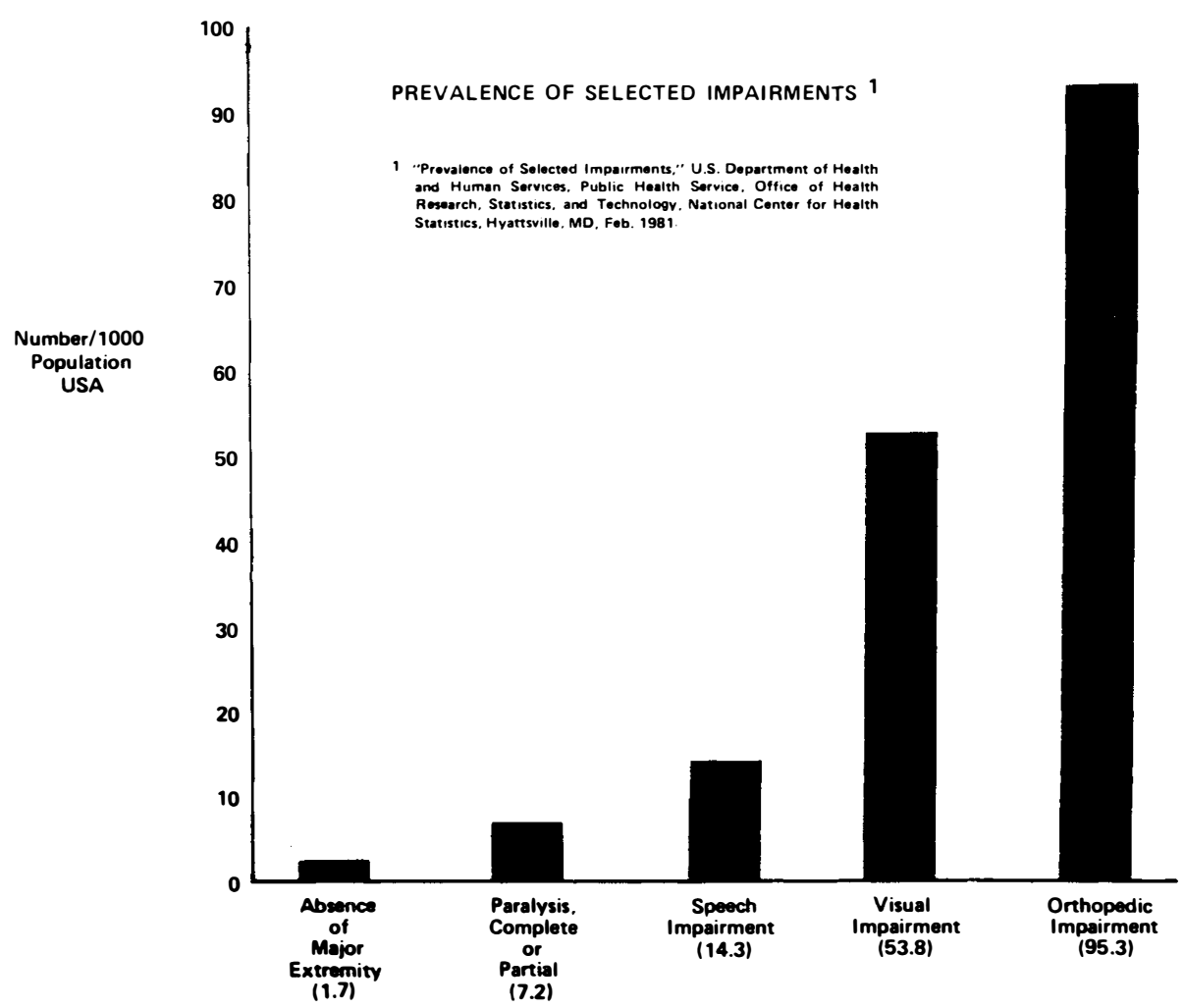

FIG. I

Prevalence of selected impairments.

\section{Catheter insertion mirror}

Need

Self-insertion by females is complicated by their inability to see the point of insertion of the catheter.

\section{Solution}

A suction-cup-mounted bracket with a mirror that can be attached to the commode at the proper position and angle is shown in Figure 3. By moving the suction cup around on the front of the commode, it is possible to adjust the angle of the mirror so that the point of insertion can be easily seen.

\section{Cost}

Most of the pieces can be bought at local stores for less than U.S. $\$ 5.00$. The labour required is approximately 30 minutes. 


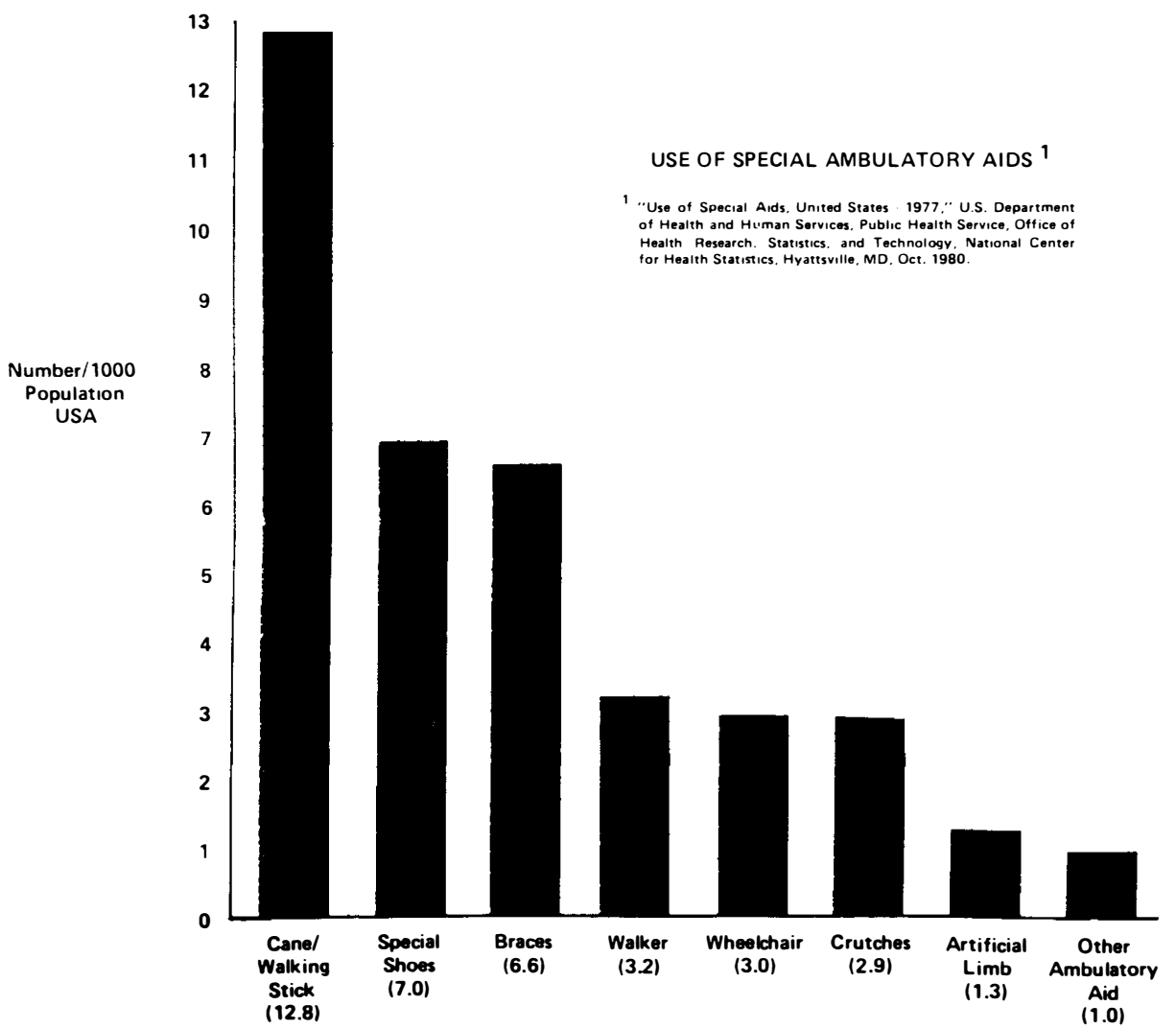

FIG. 2

Use of special ambulatory aids.

\section{Knee spreader}

Need

Knee spreaders are frequently required for wheelchair patients but the need is often only temporary, so that low cost units are very desirable.

\section{Solution}

A hot-wire cutter with two sheet metal templates was devised which permits the easy fabrication of knee spreaders to any given dimension from styrofoam (Fig. 4) which is very lightweight and of low cost. The two templates are used to obtain the cut-outs into which the legs fit. Because of its extreme light weight, it tends to stay in place and does not fall out readily.

Cost

The cutter requires approximately U.S. $\$ 25.00$ to fabricate but take care to ensure electrical safety. Approximately 2 hours labour is required to 


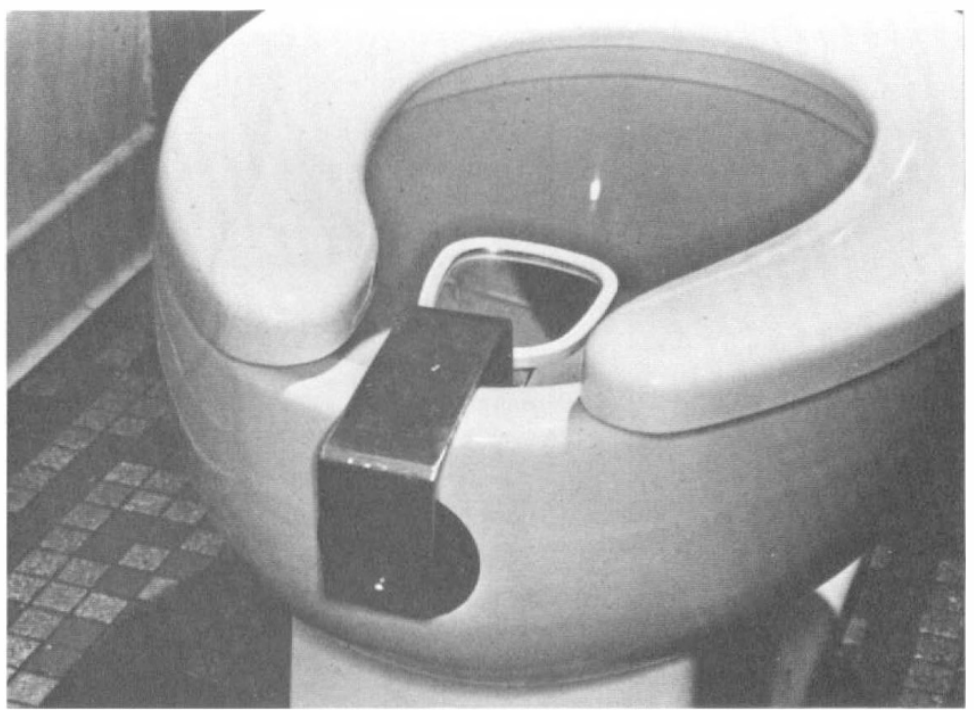

FIG. 3

Catheter insertion mirror.

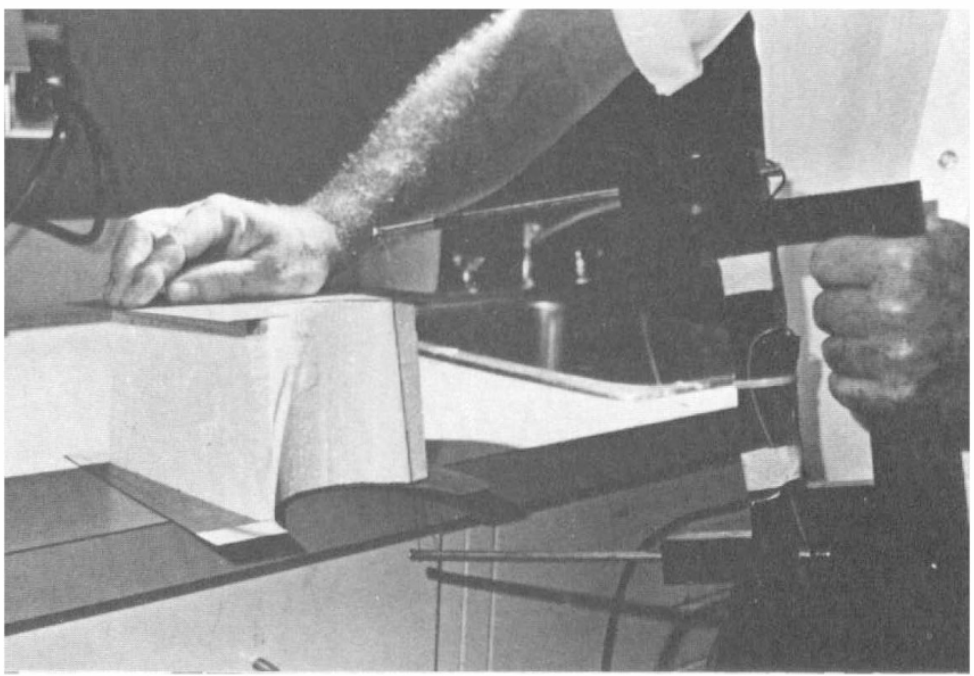

FIG. 4

Cutting styrofoam block for knee spreader.

make the cutter itself including the attachment of the nichrome wire. Individual knee spreaders, once the dimensions have been measured on the patient, require approximately Io to is minutes to install with a material cost of less than U.S. \$I.OO. 


\section{Parallel bars}

Need

Some patients need parallel bars for exercise at home after they leave the rehabilitation centre but unfortunately commercially available bars are quite expensive.

\section{Solution}

We have elected to use a stand-alone parallel bar system. An alternative which might be cheaper, would be to bolt one end of the parallel bar to the wall of the building so that only one set of support legs would be required. (Figs 5 and 6).

\section{SIDE VIEW}

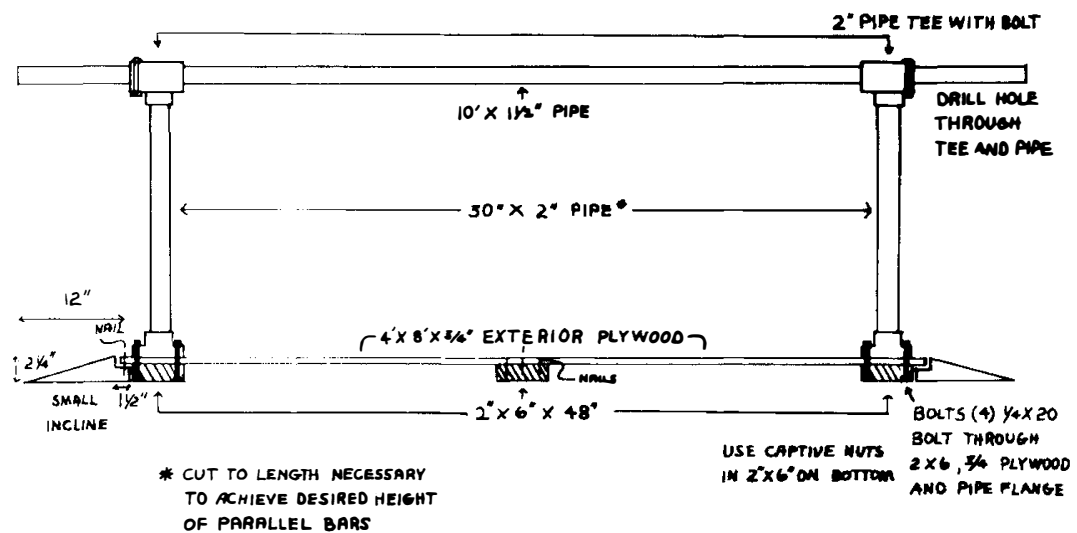

FIG. 5

Parallel bars, side view.

\section{END VIEW}

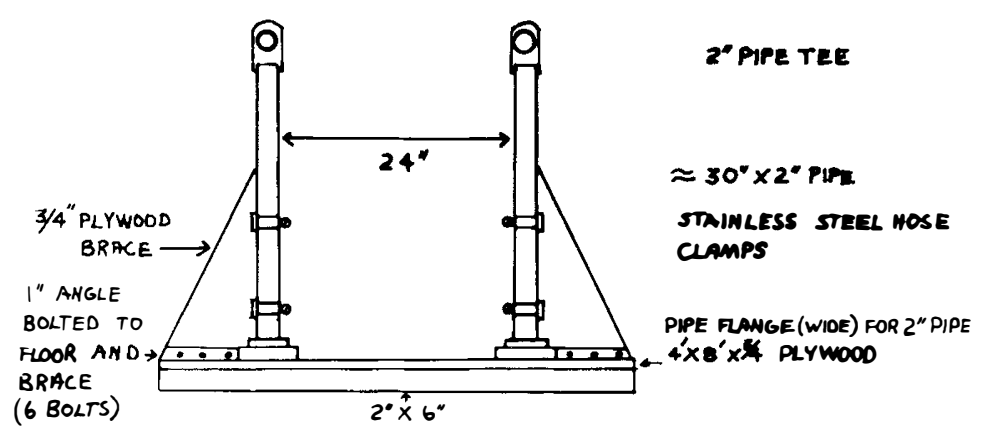

FIG. 6

Parallel bars, end view 
Cost

The materials are galvanised pipe and pipe fittings and exterior plywood plus several wooden stiffener members for the floor of the parallel bars. The side braces, shown in Figure 6, prevent spreading of the bars when weight is applied to the top. For very lightweight people, side braces may not be necessary. The cost is approximately U.S. \$I65.00, and 4 to 8 hours of unskilled time for the assembly.

\section{Rolling arm supports}

Need

Rolling arm supports are primarily intended for use by arthritic patients who are not able to use conventional parallel bars. The rolling arm support is primarily used at MMRC for arthritic patients having wrist, hand, and/or elbow involvement to allow a much smoother gait.

\section{Solution}

Supports were fabricated using conduit, ball bearings, four small sections of pipe, and a set of forearm extensions used on walkers.

\section{Cost}

Materials are approximately U.S. \$235.00. Labour requires approximately 8 hours with access to a welding torch, a drill press, and a pipe cut-off machine desirable. A more detailed description of the sliding forearm rest for parallel bars has been published separately (Posey \& Pearce, I983).

\section{Car door openers}

Need

An inexpensive car door opener for newer car doors.

\section{Solution}

This design has been used quite successfully, particularly with modern General Motors cars. The door opener is constructed from a piece of conduit with an appropriately bent flat steel bar welded to it. A piece of heatshrink plastic tubing can be applied to the upper tip of the door opener to prevent scratching or rubbing of the handle during opening of the door.

Cost

Materials cost for the door opener is less than U.S. \$1.00. The labour required is approximately 30 minutes. A welding machine is required to attach the metal strap to the conduit, and a vice is desirable to accomplish the bending of the metal strap. 


\section{Hand grip for forearm crutches}

Need

Heavy patients attempting to use conventional forearm crutches cause tissue trauma to their hands because of high pressure concentrations.

Primarily because of the geometry of the handle on commercially available forearm crutches, heavy patients frequently encounter high pressures and tissue trauma in the palm of the hand during use of the crutches. It should be noted that most patients are not heavy enough and do not use the crutches often enough to find this to be a problem.

\section{Solution}

A contoured and angled handgrip can be fabricated using wood, foam padding, and a vinyl cover. Figure 7 shows a cross-sectional side view of the modifications necessary to the handgrip of forearm crutches. A wooden base is attached to the forearm crutch handgrip. This wooden platform is angled so as to provide the proper ergonomic fit to the hand of the patient. The wooden piece is attached by means of bolts. One-half inch of high density foam is glued to the top surface of the wood, and then the entire surface of the unit is covered with a soft leather. Figure 8 shows the modified handgrip in its completed form. There are three reasons why the pressure and corresponding tissue trauma are reduced with this design. Firstly, the crutch handle is normally one-inch in diameter. Use of the wooden piece, which is one and one-half inches wide, increases the area of support by 50 per cent, thus reducing the load. Secondly, the foam rubber tends to provide some equalisation of pressure and cushioning, again

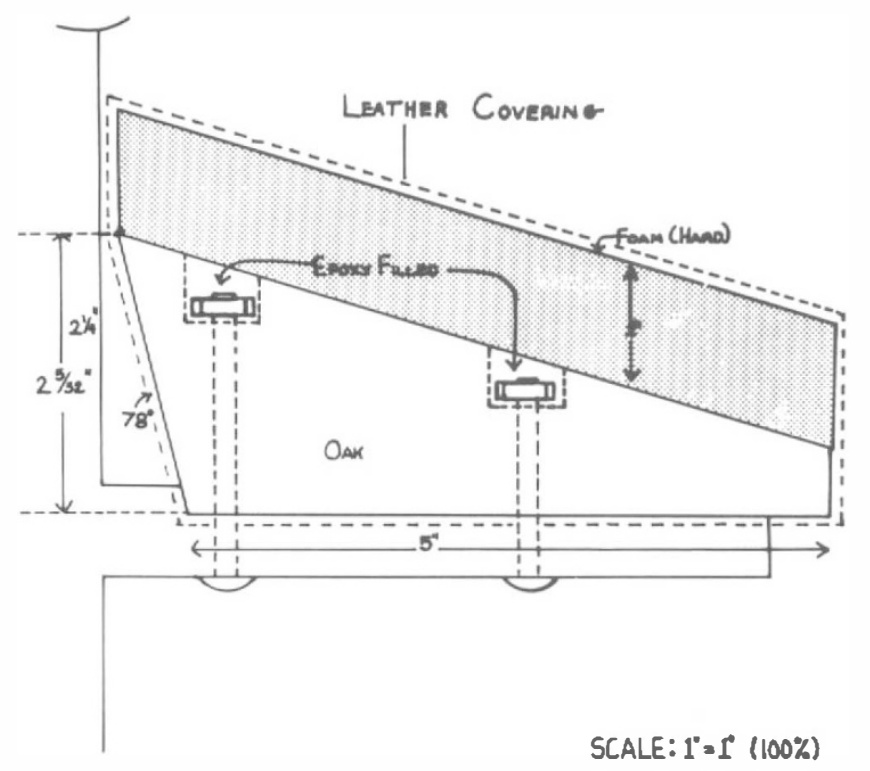

FIG. 7

Hand grip, cross-section. 


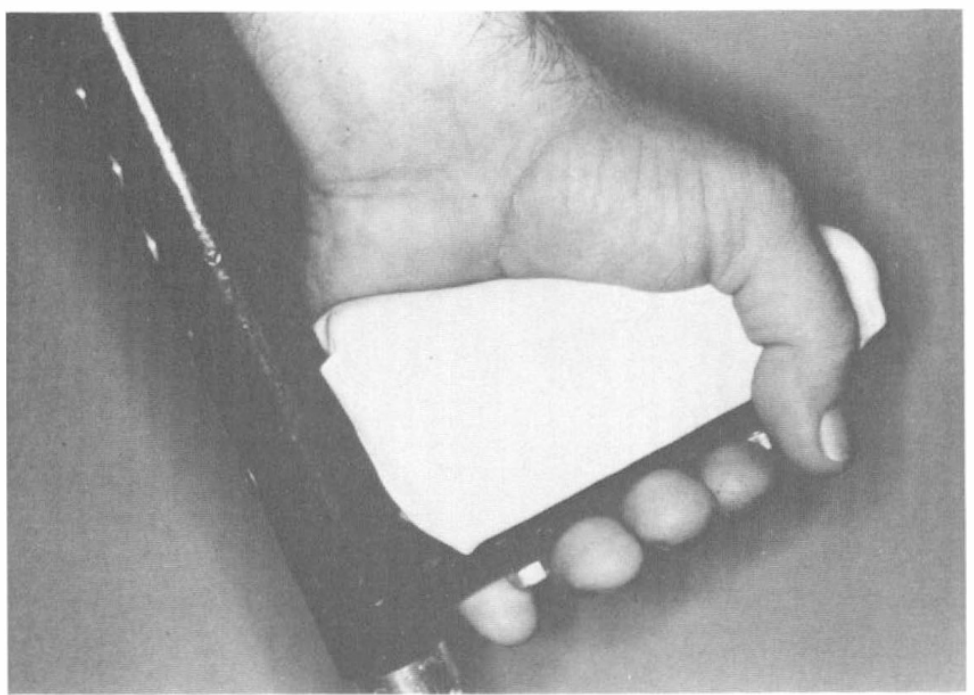

FIG. 8

Hand grip installed in forearm crutch.

reducing pressures. Finally, by providing a handle that is in the proper anatomical position for use of the crutches, the loading is even more significantly reduced. The angle shown is chosen to achieve maximum surface area of contact of the palm of the hand with the handgrip. Particular emphasis is placed on obtaining weightbearing in the fleshy part of the hand as opposed to the bony parts of the hand, as is the case with the conventional handgrip.

Cost

The materials cost on these hand grips is less than U.S. \$5.00. Approximately 2 hours labour is required to fabricate both handgrips.

\section{Puff nurse call}

Need

Tetraplegic patients who are unable to use conventional hand-operated nurse call buttons need an easily fabricated, highly reliable puff or suck pressure switch to interface with nurse call systems.

\section{Solution}

An adjustable, commercial air switch with flexible vinyl tubing and parts from local electronics suppliers is completely satisfactory, easily used, and highly reliable. It presents a switch closure or switch opening as desired to the nurse call in response to a puff or a suck on a plastic tube by the patient. Figure 9 shows the puff nurse call attached to a hospital bed. 


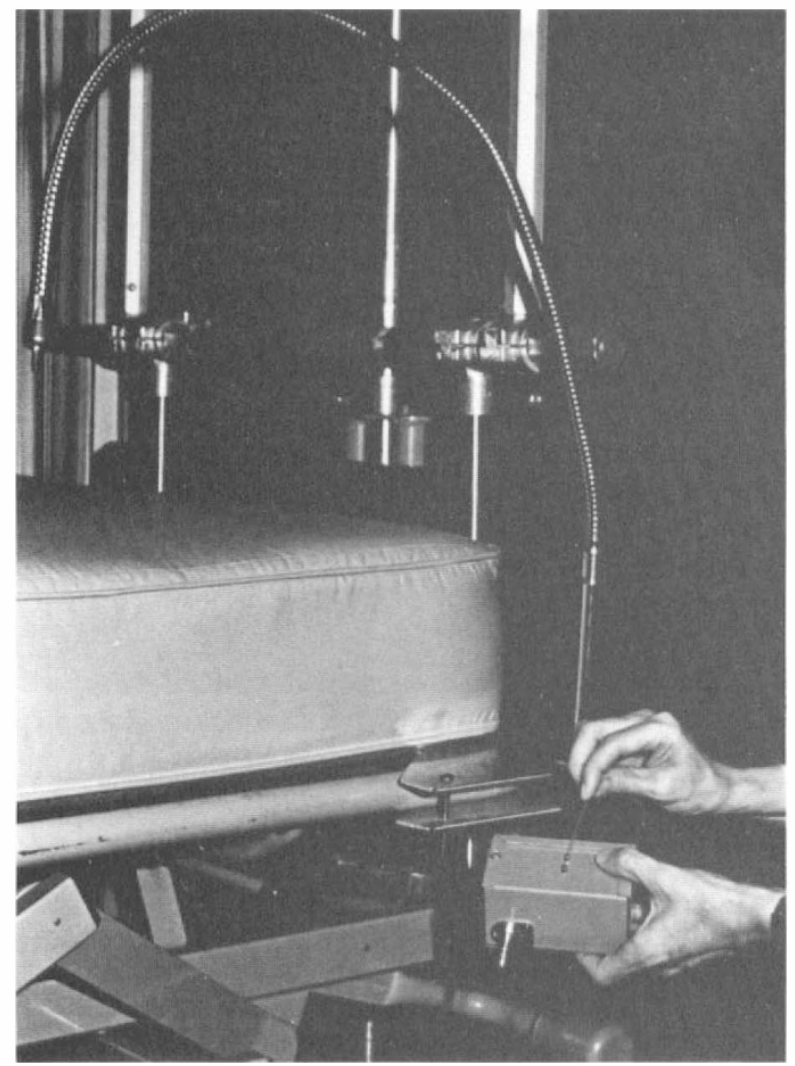

FIG. 9

Nurse call.

In the event some other type of bed is used, it is only necessary to modify the bracket holding the gooseneck to the bed. The gooseneck, which has a flexible piece of vinyl tubing running through it, is positioned so that the patient, by slight head movement, is able to place his lips on the end of the vinyl tube. The other end of the vinyl tube is connected to a unit shown in detail in Figure Io. The length of the vinyl tubing connecting from the bed-mounted apparatus to the small grey box which is plugged into the nurse call system can be several feet long. Extraordinarily long lengths of tubing are undesirable because of the fact that many patients who use such systems are not able to generate a significant volume of air with which to puff or suck. The support for the tube which is attached to the bed consists of two lengths of flexible microphone support that can be purchased from local radio supply houses.

A nipple on the end consists of a copper plumbing nipple with hole drilled in it and a 0.25 inch copper tubing soldered therein. A piece of plastic tubing is connected to the 0.25 inch copper tubing and run through the gooseneck microphone support all the way to the grey box which contains the switch. The gooseneck microphone supports are attached to the bed bracket which supports the entire apparatus. The grey box, 


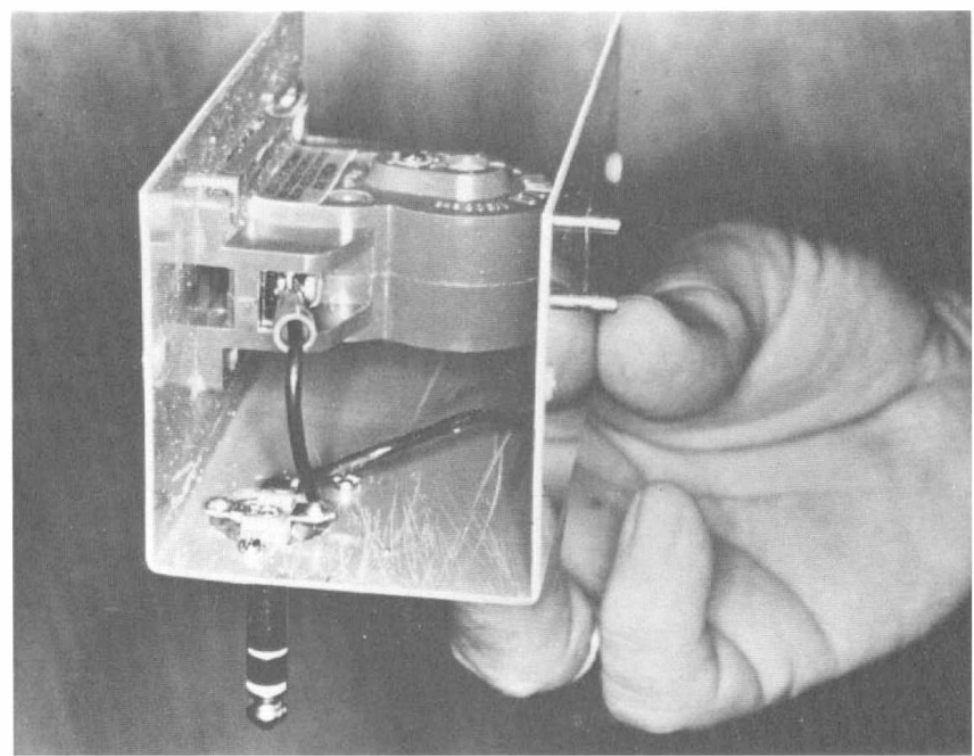

Fig. IO

Pneumatic (air wave) switch.

shown in Figure Io with the cover removed, consists of an 'air wave' switch to which the vinyl tubing is connected. The electrical contacts of the switch are connected to a phone jack or other interface connector to the nurse call system. The air wave switch is a rather expensive switch, and there are many cheaper switches available. This particular unit, however, has demonstrated extremely high reliability in applications in our hospital. Some of these units have been in continuous use for eight years without a single failure.

Cost

Approximately U.S. $\$ 75.00$ worth of materials are required. The primary cost is the air wave switch itself. It is approximately U.S. \$35.00. Two to three hours labour are required to fabricate and assemble the various components.

\section{Foot control for electric wheelchairs}

Need

An inexpensive modification to permit control of an electric wheelchair by foot movement has been required by several of our patients.

\section{Solution}

The foot control which we have devised uses the basic hand control (joystick) supplied with the wheelchair. An extender cable is inserted in 


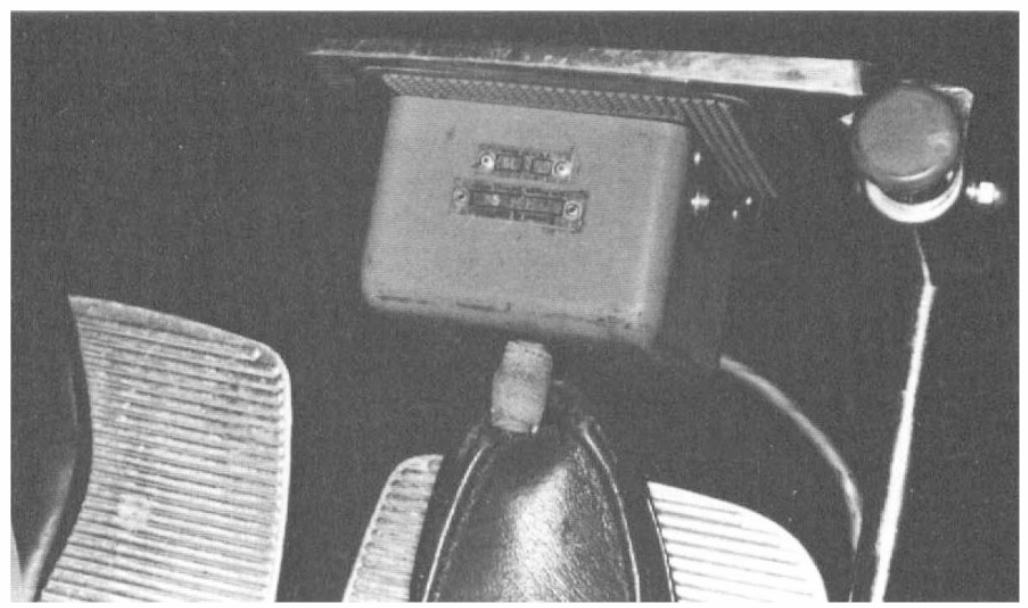

FIG. I I

Foot control.

series to permit reaching the footplate of the wheelchair. It was found convenient to use another wheelchair footplate to connect into the footplate of the wheelchair as the support platform for the control box. (Fig. I I). In order to accomplish this modification no electrical or electronic experience is required except the ability to solder and lengthen the control cable to the control box.

\section{Feedback knee goniometer}

Need

A variety of patients have gait abnormalities which result in hyperextension of the knee. A device was needed to detect hyperextension or to encourage pre-selected amount of knee flexion/extension following injury or surgery.

\section{Solution}

A lightweight knee goniometer with audio feedback and a preselectable range of actuation was designed. The feedback knee goniometer (Fig. I 2) consists of three pieces. The upper piece, which attaches by means of a belt clip, consists of a solid-state audio buzzer and a nine-volt transistor battery with holder. A cable with a quick disconnect is connected to a microswitch positioned on the middle unit on the thigh which is held in place by two velcro straps. The lower unit consists of a base with a single velcro strap and a flexible plastic. The bar is fitted into the middle unit, which has a flattened channel which entraps the plastic bar so that, as the patient flexes the knee, the bar travels in the channel corresponding to the degree of knee flexion. In use, the alarm is attached to the patient's belt or clothing, the thigh unit is put in place, then the knee is positioned to the point beyond which alarm is required for 


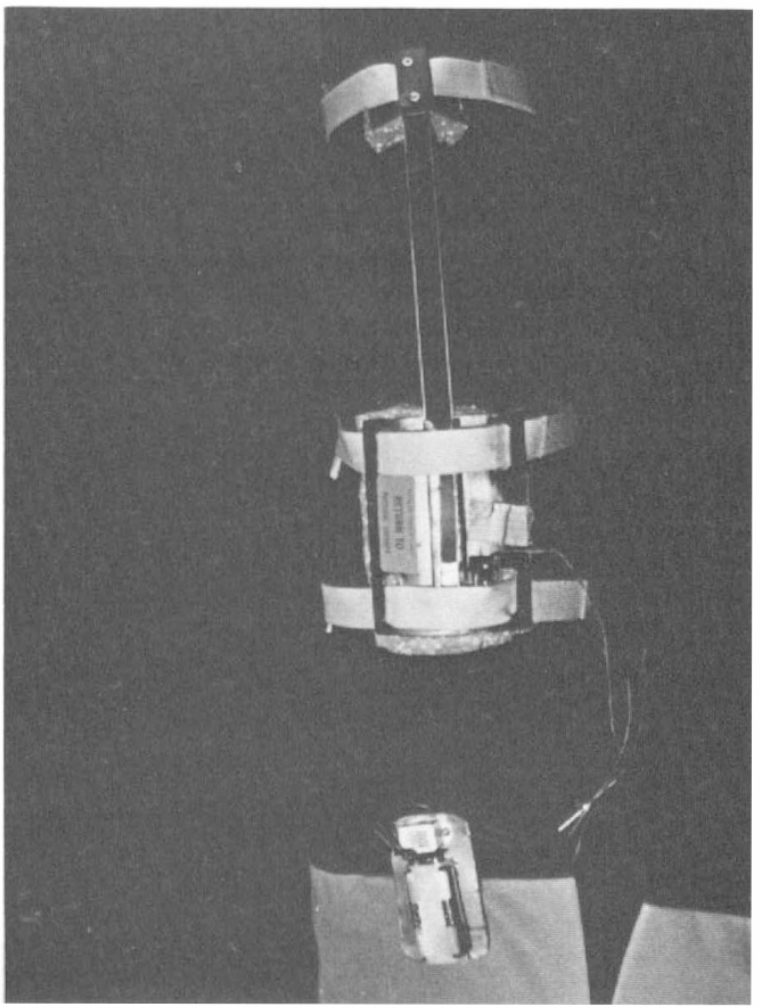

FIG. I 2

Feedback knee goniometer, side view.

hyperextension. The lower unit is then positioned so that the plastic bar just barely causes the alarm to sound. The patient then ambulates, and if he exceeds the pre-set amount of hyperextension the alarm will sound providing him with audio feedback.

\section{Cost}

At a cost of less than U.S. $\$ 20.00$, the amount of labour required to fabricate the unit was approximately one to two hours.

\section{REFERENCE}

Posey, S. \& Pearce, D. H. (1983). Sliding forearm rest for parallel bars. F. Physical Therapy, 63, 347-348. 